\title{
ANALYSIS OF PHASE SPACE STRUCTURE FOR MATCHED INTENSE CHARGED-PARTICLE BEAMS IN PERIODIC FOCUSING TRANSPORT SYSTEMS*
}

\author{
Renato Pakter, ${ }^{1}$ Chiping Chen ${ }^{1}$ and Ronald C. Davidson ${ }^{2}$ \\ ${ }^{1}$ MIT Plasma Science and Fusion Center, Cambridge, MA 02139, USA \\ ${ }^{2}$ Princeton Plasma Physics Laboratory, Princeton, NJ 08543, USA
}

\section{Abstract}

Test particle motion is analyzed for a matched intense charged-particle beam in a periodic focusing solenoidal magnetic field to assess the effects of beam intensity on inducing chaotic particle motion and halo formation.

\section{INTRODUCTION}

Halo formation and control in intense charged-particle beams has been the subject of recent vigorous theoretical, computational and experimental investigations [1]. It is of fundamental importance in the development of nextgeneration high-intensity accelerators for basic scientific research in high-energy and nuclear physics as well as for a wide variety of applications ranging from heavy ion fusion, accelerator production of tritium, accelerator transmutation of nuclear waste, spallation neutron sources, and high-power free-electron lasers. In these high-intensity accelerators, beam halos must be controlled in order to minimize beam losses and activation of the accelerator structure.

\section{THEORETICAL MODEL}

We consider a thin, continuous, intense charged-particle beam propagating in the $z$-direction with characteristic axial velocity $\beta_{b} c$ and kinematic energy $\gamma_{b} m c^{2}$ through the periodic focusing solenoidal magnetic field

$$
\mathbf{B}^{s o l}(\mathbf{x})=B_{z}(s) \mathbf{e}_{z}-\frac{1}{2} B_{z}^{\prime}(s)\left(x \mathbf{e}_{x}+y \mathbf{e}_{y}\right) .
$$

Here, $\mathbf{e}_{x}$ and $\mathbf{e}_{y}$ are unit Cartesian vectors perpendicular to the beam propagation direction, $s=z$ is the axial coordinate, $x \mathbf{e}_{x}+y \mathbf{e}_{y}$ is the transverse displacement from the beam axis at $(x, y)=(0,0)$, the superscript 'prime' denotes $d / d s$ with $B_{z}^{\prime}(s)=d B_{z}(s) / d s$, and the axial component of magnetic field satisfies

$$
B_{z}(s+S)=B_{z}(s)
$$

where $S$ is the axial period of the focusing field.

To determine the self-electric and self-magnetic fields consistently, we make the following assumptions: (a) the

* This research was supported by Department of Energy under Grant No. DE-FG02-95ER-40919 and Contract No. DE-AC02-76-CH0-3073, and by Air Force Office of Scientific Research under Grant No. F4962097-1-0325. The research by R. Pakter was also supported by CAPES, Brazil.
Budker parameter $\nu=N_{b} q^{2} / m c^{2}$ for the beam is small compared with $\gamma_{b}$; (b) the axial momentum spread of the beam particles is small in comparison with $\gamma_{b} m \beta_{b} c$; (c) the beam is axisymmetric $(\partial / \partial \theta=0)$; and $(d)$ the beam is perfectly matched into the focusing field with uniform density profile over the beam cross section,

$$
n_{b}(r, s)= \begin{cases}N_{b} / \pi r_{b}^{2}(s), & 0 \leq r<r_{b}(s), \\ 0, & r>r_{b}(s) .\end{cases}
$$

In Eq. (3), $r=\left(x^{2}+y^{2}\right)^{1 / 2}$ is the radial coordinate, $r_{b}(s)=r_{b}(s+S)$ is the outer envelope of the beam, and $N_{b}=2 \pi \int_{0}^{\infty} n_{b} r d r=$ const. is the number of particles per unit axial length. The periodic outer beam envelope $r_{b}(s)=r_{b}(s+S)$ corresponds to a special solution of the beam envelope equation $[2,3]$

$$
\frac{d^{2} r_{b}}{d s^{2}}+\kappa_{s}(s) r_{b}-\frac{K}{r_{b}}-\frac{\varepsilon_{T}^{2}}{r_{b}^{3}}=0
$$

where $K=2 q^{2} N_{b} / \gamma_{b}^{3} \beta_{b}^{2} m c^{2}$ is the normalized perveance, $\kappa_{z}(s)=\left[q B_{z}(s) / 2 \gamma_{b} \beta_{b} m c^{2}\right]^{2}=\left[\Omega_{c}(s) / 2 \beta_{b} c\right]^{2}$ is the focusing parameter, $\varepsilon_{T}=$ const. is the total unnormalized emittance, $q$ and $m$ are the particle charge and rest mass, respectively, and $c$ is the speed of light in vacuo. The transverse phase-space distribution that self-consistently generates the density profile in Eq. (3) is the rigid-rotor Vlasov equilibrium distribution with angular rotation velocity $\omega_{b}$ [2].

In cylindrical coordinates $(r, \theta)$ in the Larmor frame, the equations of motion transverse to the direction of beam propagation can be derived from the normalized Hamiltonian

$H_{\perp}\left(r, P_{r}, P_{\theta}, s\right)=\frac{1}{2}\left(P_{r}^{2}+\frac{P_{\theta}^{2}}{r^{2}}\right)+\frac{1}{2} \kappa_{z}(s) r^{2}+\psi(r, s)$,

where the normalized self-field potential $\psi(r, s)$ is defined by

$$
\psi(r, s)=\left\{\begin{array}{cc}
\frac{K}{2}\left[1-r^{2} / r_{b}^{2}(s)\right]+K \ln \left[r_{w} / r_{b}(s)\right], \\
& 0 \leq r<r_{b}(s), \\
K \ln \left[r_{w} / r\right], & r_{b}(s)<r \leq r_{w} .
\end{array}\right.
$$

\section{ANALYSIS}

In this section, we analyze the particle motion in the Larmor frame described by Eq. (5). For present purposes, the Hamiltonian in Eq. (5) is expressed as

$$
H_{\perp}\left(r, P_{r}, P_{\theta}, s\right)=H_{0}\left(r, P_{r}, P_{\theta}\right)+H_{1}\left(r, P_{r}, P_{\theta}, s\right),
$$


where

$$
\begin{gathered}
H_{0}=\frac{1}{2} P_{r}^{2}+V_{0}\left(r, P_{\theta}\right) \\
\equiv \frac{1}{2} P_{r}^{2}+\frac{1}{2} \bar{\kappa}_{z} r^{2}+\frac{P_{\theta}^{2}}{2 r^{2}}+\left.\psi(r, s)\right|_{r_{b}(s)=\bar{r}_{b}}, \\
H_{1}=\frac{1}{2}\left[\kappa_{z}(s)-\bar{\kappa}_{z}\right] r^{2}+\psi(r, s)-\left.\psi(r, s)\right|_{r_{b}=\bar{r}_{b}} .
\end{gathered}
$$

In Eqs. (7)-(9), $\psi(r, s)$ is defined in Eq. (6), and the effective mean beam radius $\bar{r}_{b}$ is defined by

$$
\bar{r}_{b}=\left(\varepsilon_{T} S / \sigma\right)^{1 / 2},
$$

where $\sigma=\varepsilon_{T} \int_{s}^{s+S} d s / r_{b}^{2}(s)$ is the space-chargedepressed phase advance for the rigid-rotor Vlasov equilibrium. The effective mean focusing parameter $\bar{\kappa}_{z}$ occuring in Eqs. (8) and (9) is defined by

$$
\bar{\kappa}_{z}=\frac{K}{\bar{r}_{b}^{2}}+\frac{\varepsilon_{T}^{2}}{\bar{r}_{b}^{4}} .
$$

Physically, the Hamiltonian $H_{0}$ provides a good approximate description of the (slow) betatron oscillations, whereas the perturbation $H_{1}$ describes nonlinear resonances induced by the (fast) oscillations in $\kappa_{z}(s)$ and $r_{b}(s)$.

To determine the betatron oscillation frequency, we employ the Hamilton-Jacobi method and perform a canonical transformation from $\left(r, P_{r}\right)$ to the action-angle variables $(\phi, J)$. Let $W(r, J)$ be the characteristic function satisfying the partial differential equation

$$
\frac{1}{2}\left(\frac{\partial W}{\partial r}\right)^{2}+V_{0}\left(r, P_{\theta}\right)=H_{0}=\text { const. }
$$

As discussed below, the dependence of $W$ on $J$ is uniquely determined because of the one-to-one correspondence between $H_{0}$ and $J$ [see Eq. (16)]. A formal expression for the angle variable $\phi$ is given by

$$
\phi=\partial W / \partial J .
$$

The action variable $J$ can be expressed as

$$
J=\frac{1}{2 \pi} \oint P_{r} d r=\frac{1}{\pi} \int_{r_{-}}^{r_{+}}\left\{2\left[H_{0}-V_{0}\left(r, P_{\theta}\right)\right]\right\}^{1 / 2} d r,
$$

where the turning points $r_{ \pm}$solve the algebraic equation

$$
H_{0}=V_{0}\left(r_{ \pm}, P_{\theta}\right),
$$

and $r_{+}>r_{-}$is assumed. Because the action variable $J$ increases monotonically with increasing $H_{0}$, Eq. (14) can be inverted to yield a Hamiltonian of the form

$$
H_{0}=H_{0}\left(J, P_{\theta}\right) .
$$

The betatron oscillation frequency can then be expressed as

$$
\omega_{\beta}\left(J, P_{\theta}\right)=\partial H_{0} / \partial J
$$

which, in general, must be evaluated numerically.

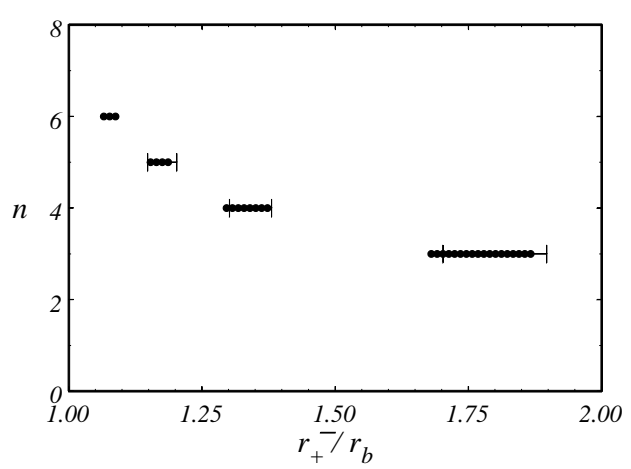

Figure 1: Locations and full widths of the primary resonances of order $n=3$ to 6 .

Under the influence of the perturbation $H_{1}$, a variety of nonlinear resonances occur due to the coupling of the (slow) betatron oscillations and the (fast) oscillations in the focusing parameter $\kappa_{z}(s)$ and associated modulation in the beam envelope $r_{b}(s)$. The locations and widths of the nonlinear resonances are analyzed.

Making use of the action-angle variables $(\phi, J)$, we express the total Hamiltonian $H$ formally as

$$
H\left(\phi, J, P_{\theta}, s\right)=H_{0}\left(J, P_{\theta}\right)+H_{1}(\phi, J, s) .
$$

Expanding $H_{1}$ in a Fourier series representation in $\phi$ and $s$, we obtain

$$
H_{1}=\sum_{n=-\infty}^{\infty} \sum_{l=-\infty}^{\infty} a_{n l}(J) \exp [i(n \phi+2 l \pi s / S)],
$$

where the Fourier coefficients $a_{n l}(J)$ are determined numerically. Of particular interest in the present analysis are the primary nonlinear resonances that satisfy the resonance condition

$$
n \omega_{\beta}\left(J_{n}, P_{\theta}\right)=2 \pi / S,
$$

where $J_{n}$ determines the location of the primary resonance of order $n$ in the phase space $(\phi, J)$, i.e., at $J=J_{n}$. The full width of the $n$ th-order primary resonance is estimated to be

$$
\Delta r_{n}=\left(\frac{\partial r}{\partial J}\right)_{P_{\theta}, J=J_{n}}\left[\frac{32\left|a_{n,-1}\left(J_{n}\right)\right|}{\left(\partial \omega_{\beta} / \partial J\right)_{J=J_{n}}}\right]^{1 / 2}
$$

in the radial coordinate.

Figure 1 shows the locations and full widths of the primary resonances of order $n=3$ to 6 obtained for the choice of system parameters corresponding to $\sigma_{v}=80^{\circ}$ $\left(S^{2} \hat{\kappa}_{z}=8.712\right), \eta=0.2, \sigma=26.2^{\circ}\left(S K / \varepsilon_{T}=3.8\right)$, $\omega_{b}=0$ and $P_{\theta}=0$. Here, a step-function lattice is used, and $\eta$ is the filling factor. In Fig. 1, the solid lines correspond to the analytical estimates given in Eq. (21), whereas the dotted lines are obtained by integrating Eq. (5) numerically.

Use is made of the Poincaré surface-of-section method to examine extensively the phase-space structure described 


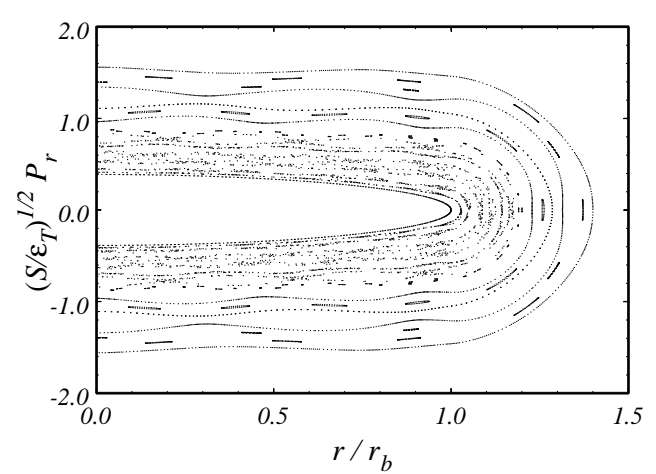

Figure 2: Poincaré surface-of-section plots in the phase space $\left(r, P_{r}\right)$ for 15 test particle trajectories moving through the periodic step-function lattice from $s / S=0.5$ to 1000.5. Here, the parameters are: $\sigma_{v}=80^{\circ}, \eta=0.2$, $\sigma=11.4^{\circ}\left(S K / \varepsilon_{T}=10\right), \omega_{b}=0$, and $P_{\theta} / \varepsilon_{T}=0$.

by the Hamiltonian $H$ in Eq. (5). Of particular interest are the nonlinear resonances and chaotic particle motion of test particles outside the boundary of the phase space occupied by the interior beam particles making up the rigid-rotor Vlasov equilibrium distribution $f_{0}$ [2]. The phase-space boundary of the rigid-rotor Vlasov equilibrium is a closed surface in the three-dimensional phase space $\left(r, P_{r}, P_{\theta}\right)$ at any given axial distance $s$. A projection of such a boundary onto the phase space $\left(r, P_{\theta}\right)$ can be determined from

$$
\left[\frac{P_{\theta} r_{b}(s)}{\varepsilon_{T} r}+\omega_{b} \frac{r}{r_{b}(s)}\right]^{2}+\left(1-\omega_{b}^{2}\right) \frac{r^{2}}{r_{b}^{2}(s)}=1-\omega_{b}^{2}
$$

where the parameter $\omega_{b}\left(\left|\omega_{b}\right|<1\right)$ is a measure of beam rotation in the Larmor frame. For a KV equilibrium distribution, $\omega_{b}=0$.

Detailed results of the phase-space analysis are discussed in [4], and are summarized in Sec. 4. Here, we only illustrate the effect of beam rotation on beam dynamics in Figs. 2 and 3. For both cases shown in Figs. 2 and 3 , the value of $P_{\theta}$ is chosen such that the boundary of the equilibrium distribution extends to $r=r_{b}$ [Eq. (22)]. It is evident in Figs. 2 and 3 that, for comparable choices of system parameters, the phase space structure for a nonrotating $\mathrm{KV}$ equilibrium distribution $\left(\omega_{b}=0\right)$ exhibits more pronounced chaotic behavior that that for a rigid-rotor Vlasov equilibrium distribution (with $\omega_{b}=0.9$ ).

\section{CONCLUSIONS}

Test particle motion has been analyzed for matched intense charged-particle beam propagating through a periodic solenoidal magnetic field. The betatron oscillations of test particles in the average self fields and applied field were analyzed, and the nonlinear resonances induced by periodic modulations in the self fields and applied field were determined. It was found [4] that the phase-space structure changes significantly as the canonical angular momentum

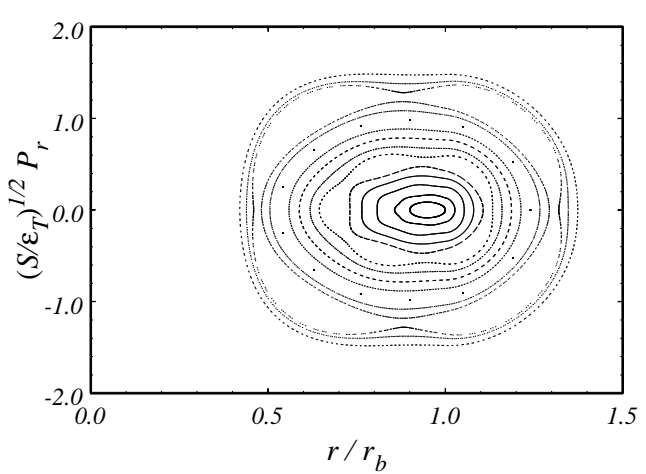

Figure 3: Poincare surface-of-section plots in the phase space $\left(r, P_{r}\right)$ for 15 test particle trajectories moving through the periodic step-function lattice from $s / S=0.5$ to 1000.5. Here, the parameters are: $\sigma_{v}=80^{\circ}, \eta=0.2$, $\sigma=11.4^{\circ}\left(S K / \varepsilon_{T}=10\right), \omega_{b}=0.9$, and $P_{\theta} / \varepsilon_{T}=-0.9$.

$\left(P_{\theta}\right)$, beam intensity (as measured by $S K / \varepsilon_{T}$ or $\sigma / \sigma_{v}$ ), vacuum phase advance $\sigma_{v}$, or beam rotation $\left(\omega_{b}\right)$ is varied. For an intense beam with nonrotating KV equilibrium distribution $\left(\omega_{b}=0\right)$, it was shown that the chaotic regions approach the phase-space boundary of the equilibrium distribution as the canonical angular momentum $P_{\theta}$ decreases in magnitude. For an intense beam with a rigid-rotor Vlasov equilibrium distribution $\left(\omega_{b} \neq 0\right)$, it was found that the presence of beam rotation reduces the degree of chaotic behavior in phase space. Finally, for $\sigma_{v}<80^{\circ}$, the testparticle analysis showed that at very high beam intensities, the chaotic layers associated with the separatrices of nonlinear resonances are still divided by the remaining invariant (KAM) surfaces and do not overlap completely to form an extended chaotic region.

\section{REFERENCES}

[1] American Institute of Physics Conf. Proc. 377, edited by S. Y. Lee (1996).

[2] C. Chen, R. Pakter, and R. C. Davidson, Phys. Rev. Lett. 79, 225 (1997).

[3] R. C. Davidson and C. Chen, Part. Accel. 59, 175 (1998).

[4] C. Chen, R. Pakter, and R. C. Davidson, submitted to Phys. Rev. E (1998). 